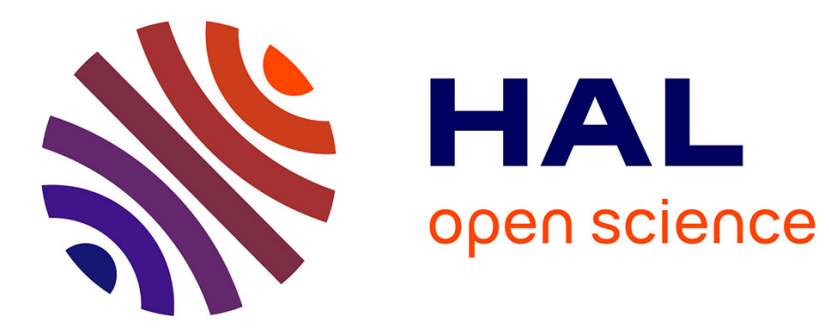

\title{
Capturing reading patterns through a real-time smart camera iris tracking system
}

Mehrube Mehrubeoglu, Evan Ortlieb, Lifford Mclauchlan, Linh Manh Pham

\section{To cite this version:}

Mehrube Mehrubeoglu, Evan Ortlieb, Lifford Mclauchlan, Linh Manh Pham. Capturing reading patterns through a real-time smart camera iris tracking system. SPIE 8437, Real-Time Image and Video Processing 2012, 843705, Jun 2012, Brussels, Belgium. 10.1117/12.922875 hal-01233270

\section{HAL Id: hal-01233270 \\ https://hal.science/hal-01233270}

Submitted on 24 Nov 2015

HAL is a multi-disciplinary open access archive for the deposit and dissemination of scientific research documents, whether they are published or not. The documents may come from teaching and research institutions in France or abroad, or from public or private research centers.
L'archive ouverte pluridisciplinaire $\mathbf{H A L}$, est destinée au dépôt et à la diffusion de documents scientifiques de niveau recherche, publiés ou non, émanant des établissements d'enseignement et de recherche français ou étrangers, des laboratoires publics ou privés. 


\title{
Capturing reading patterns through a real-time smart camera iris tracking system
}

\author{
Mehrube Mehrubeoglu*a ${ }^{*}$ Evan Ortlieb ${ }^{\mathrm{a}}$, Lifford $\mathrm{M}^{\mathrm{c}}$ Lauchlan $^{\mathrm{b}}$ and Linh M. Pham ${ }^{\mathrm{a}}$ \\ ${ }^{a}$ Texas A\&M University-Corpus Christi, 6300 Ocean Dr., Corpus Christi, TX, USA, 78412-5797 \\ ${ }^{\mathrm{b}}$ Texas A\&M University-Kingsville, 700 University Blvd., MSC 192, Kingsville, TX, USA, 78363
}

\begin{abstract}
A real-time iris detection and tracking algorithm has been implemented on a smart camera using LabVIEW graphical programming tools. The program detects the eye and finds the center of the iris, which is recorded and stored in Cartesian coordinates. In subsequent video frames, the location of the center of the iris corresponding to the previously detected eye is computed and recorded for a desired period of time, creating a list of coordinates representing the moving iris center location across image frames. We present an application for the developed smart camera iris tracking system that involves the assessment of reading patterns. The purpose of the study is to identify differences in reading patterns of readers at various levels to eventually determine successful reading strategies for improvement. The readers are positioned in front of a computer screen with a fixed camera directed at the reader's eyes. The readers are then asked to read preselected content on the computer screen, one comprising a traditional newspaper text and one a Web page. The iris path is captured and stored in real-time. The reading patterns are examined by analyzing the path of the iris movement. In this paper, the iris tracking system and algorithms, application of the system to real-time capture of reading patterns, and representation of 2D/3D iris track are presented with results and recommendations.
\end{abstract}

Keywords: real-time iris tracking, eye tracking, smart camera, LabVIEW

\section{INTRODUCTION}

\subsection{Background}

Eye tracking has many applications including the determination of driver attention, gaze tracking, or reading patterns. Finding eye locations facilitates iris detection and tracking. Xiang and Cheng determine eye locations using improved AdaBoost algorithm. ${ }^{1} \mathrm{Xu}$ et al. determine the eye corners and iris centers by utilizing a webcam in normal lighting to obtain images. ${ }^{2}$ Sun et al. employ Hough transforms to determine driver gaze direction and driver's iris position for improving driver safety. ${ }^{3}$ Hopf, Neumann, and Przewozny compare multi-camera and HD camera systems for tracking eyes from multiple users. ${ }^{4}$ They find the multi-camera setup outperforms the HD camera system. Tan and Zhang employ edge information in addition to intensity to facilitate iris detection and to determine eye states, open/closed. ${ }^{5}$

Common methods for object tracking include the use of Kalman and particle filters. Such filters have been applied for detection and tracking eye locations ${ }^{6-9}$. Xie, Sudhakar, and Zhuang assume small eye movements in order to linearize the eye location tracking model using Kalman filters. ${ }^{6,8}$ Asano, Takano, and Nakamura utilize particle filters along with edge information to detect and track eye locations in the presence of pose changes. ${ }^{9}$ Keeping track of eye location will aid with the determination of reading patterns which can be used to increase reading proficiency.

In this paper, LabVIEW-based real-time eye detection and iris finding algorithms have been implemented on a smart camera to find and record iris center location across video frames. The system incorporates template matching and normalized cross-correlation to detect eye location based on a pre-stored eye template per user. Then, iris location is found through edge detection techniques. Iris center is computed in Cartesian coordinates and stored in a spreadsheet file for off-line analysis.

\subsection{Iris Tracking Application and Motivation}

Reading improvement is of utmost importance to college and career readiness ${ }^{10}$. In 2007, the National Assessment of Adult Literacy in the United States reported "reading scores of American Adults of all education levels have lessened

*ruby.mehrubeoglu@tamucc.edu; phone 13618253378 
especially noted among the best educated groups.” From 1992 to 2003, the proportion of U.S. college graduates who can read at a proficient level declined to $31 \%$ from a $40 \%$ level. In 2004, almost $42 \%$ of all freshmen enrolled in public 2 year colleges were enrolled in at least one developmental course ${ }^{11}$.

Some developmental reading programs incorporate technology to practice or teach the reading skills. Computer labs, either through an onsite computer lab or a virtual lab, can be accessible to students seeking reading improvement. Though studies have examined the effects of interventions, additional research is warranted to examine eye movements during the reading of digital texts to provide a conceptual framework for improving students' digital reading skills.

Good readers make fewer fixation pauses and regressions during traditional print-based text reading ${ }^{12}$. Younger readers or poor readers make a larger number of fixations and regressions in reading. This tendency changes as readers practice, learn more vocabulary words, and develop comprehension skills. Patterns of eye movements are clues to one's level of reading proficiency. Existing research has primarily been conducted in remedial programs or reading clinics related to reading disability. In turn, students were trained in eye movement exercises to improve reading performance. These studies were based on the notion if a poor reader learned good eye movement patterns, their reading would improve including fluency and comprehension.

Although comprehension is central to reading, information is processed only when the eye pauses within an eye fixation $^{13}$. By eliminating backward saccades or regressions, readers can increase the speed of visual processing and in turn, uptake of information. Still, no research has examined the nature of iris movements, more specifically, during the process of reading digital texts where assumptions of directionality and eye movement do not necessarily apply. In this pilot study, collegiate participants were issued a standard protocol, and read one electronic newspaper and one news website to plot/track their iris movements. The results from this study will inform researchers about digital reading behaviors.

In the remainder of this paper, the real-time iris tracking system is described in Section 2. The iris tracking process and algorithms are explained in Section 3. Data acquisition protocol and experiments are found in Section 4. Results are shown in Section 5. Finally, conclusions and future work are presented in Section 6.

\section{THE IRIS TRACKING SYSTEM}

\subsection{Hardware Setup}

The smart camera is directly connected to a regular computer (PC1 in Fig. 1) through an Ethernet cable in a local network. The data and control signal are sent back and forth in this cable in full-duplex form. The full-duplex form allows communication in both directions, which results in faster transmission. The computer has a Pentium $42.26 \mathrm{GHz}$ processor, an on-board $64 \mathrm{MB}$ graphics card and 1.25 GB RAM ${ }^{15}$. LabVIEW desktop interface is used to communicate with the smart camera. For reading pattern experiment, a second computer (PC2 in Fig. 1) is used to open sample texts such as a Web-based news article on a Website, or a story in a traditional newspaper style from a PDF file. The computer is in the same local network with the camera and the first computer. Test users (readers) sit in front of the second computer and look at its screen. The camera is mounted on a rack above of the screen. Position of the camera on the rack can be adjusted mechanically.

The main component of the eye tracking system is a NI 1762 smart camera with a Computar $12 \mathrm{~mm}$ lens and the Gigabit Ethernet interface ${ }^{14,15}$. The smart camera has dual processors. One is a $533 \mathrm{MHz}$ PowerPC processor (Freescale). The other is a 720MHz DSP (Texas Instruments). These dual processors permit the smart camera to process data up to four times faster when applying algorithms such as pattern matching and data matrix code reading. The camera has a 1/33 inch Sony ICX 424 AL solid-state image sensor with square pixels for the acquisition of grayscale images. In addition two separate $128 \mathrm{MB}$ memory units exist on board the camera. One memory unit is utilized for the operating system and the other is for job (data/program/spreadsheet) storage. Finally, the smart camera is equipped with a temperature sensor which shuts off at temperatures greater than $70{ }^{\circ} \mathrm{C}$ automatically, and resumes operation when a suitable operating temperature is reached again. The lens is attached to the camera through a $\mathrm{C}$ mount and allows high resolution images to be formed on the smart camera's sensor. Through the use of the lens and the aperture control, the amount of light and zoom can be adjusted. 


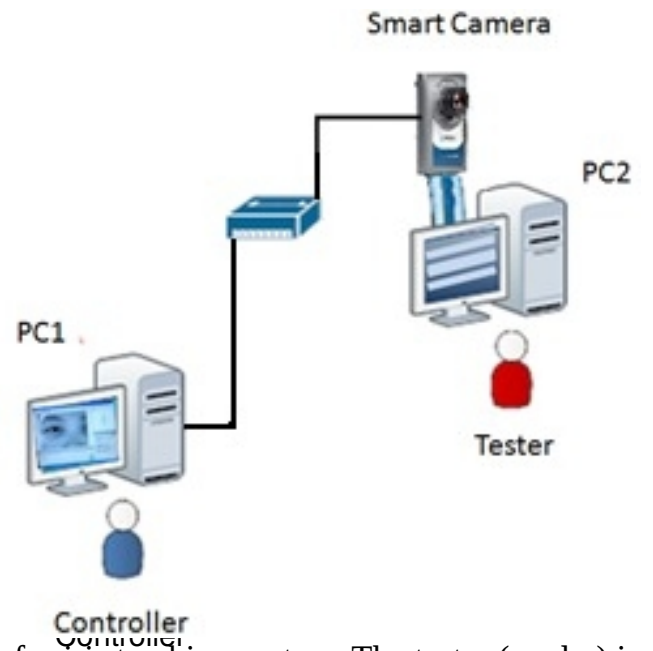

Figure 1. The experimental setup for iris tracking system. The tester (reader) is seated in front of a computer monitor with preselected text (PC2). The smart camera is positioned to capture eye images. The smart camera is connected to an independent computer (PC1) through a LAN switch and interfaced with LabVIEW-based programs for real-time iris tracking. The controller captures the user-based eye template at the beginning of the experiments, and starts and stops real-time data acquisition.

\subsection{Software}

The algorithms are developed and implemented in Vision Builder AI 3.6 that is supported by the smart camera. LabVIEW, a high-level graphical programming environment was chosen for programming and interfacing. The realtime iris tracking algorithm is implemented in LabVIEW 8.5.1, since the smart camera model does not support newer versions of LabVIEW. Connections between virtual instruments (VIs) and analog input/output devices are created under LabVIEW 8.5.1 using the G programming language. The iris tracking system requires image processing pattern recognition algorithms such as pattern matching, edge detection, and image calibration. LabVIEW provides built-in libraries useable by the NI smart camera. Virtual instruments (VIs), or interfaces, are designed and controlled via GPIB, RS232, VXI or regular Ethernet. With the camera's built-in FTP server, a FTP software, such as a FTP client can be used to connect to the internal storage of the NI smart camera directly. The TCP connection library from LabVIEW allows the smart camera to run on a local network similar to a regular computer. (The reader is referred to Mehrubeoglu et al. ${ }^{15}$ for further details on the software and implementation of algorithms.)

\section{THE IRIS TRACKING PROCESS}

In this project, real-time eye detection and iris tracking are achieved with LabVIEW-based algorithms. These algorithms are combined into embedded programs uploaded to a smart camera. Table 1 summarizes the steps involved in iris detection and tracking.

\subsection{Real-time Eye Tracking Algorithm}

Before real-time eye tracking can begin, the controller (see Fig. 1) must select and save an eye template for each user. The template selection and saving process is depicted in Figure 2. Once a single frame is captured, the controller clicks and drags the bounding box for the eye template to be saved. An eye template is selected for each reader at the beginning of the experiments. Then, the acquired video frames are analyzed to find the region with the best template match to determine the eye area in the captured frame. The camera is programmed to track the eye motion and iris center location continuously until stopped by the controller. Table 1 summarizes the steps to capture iris center location real-time. 


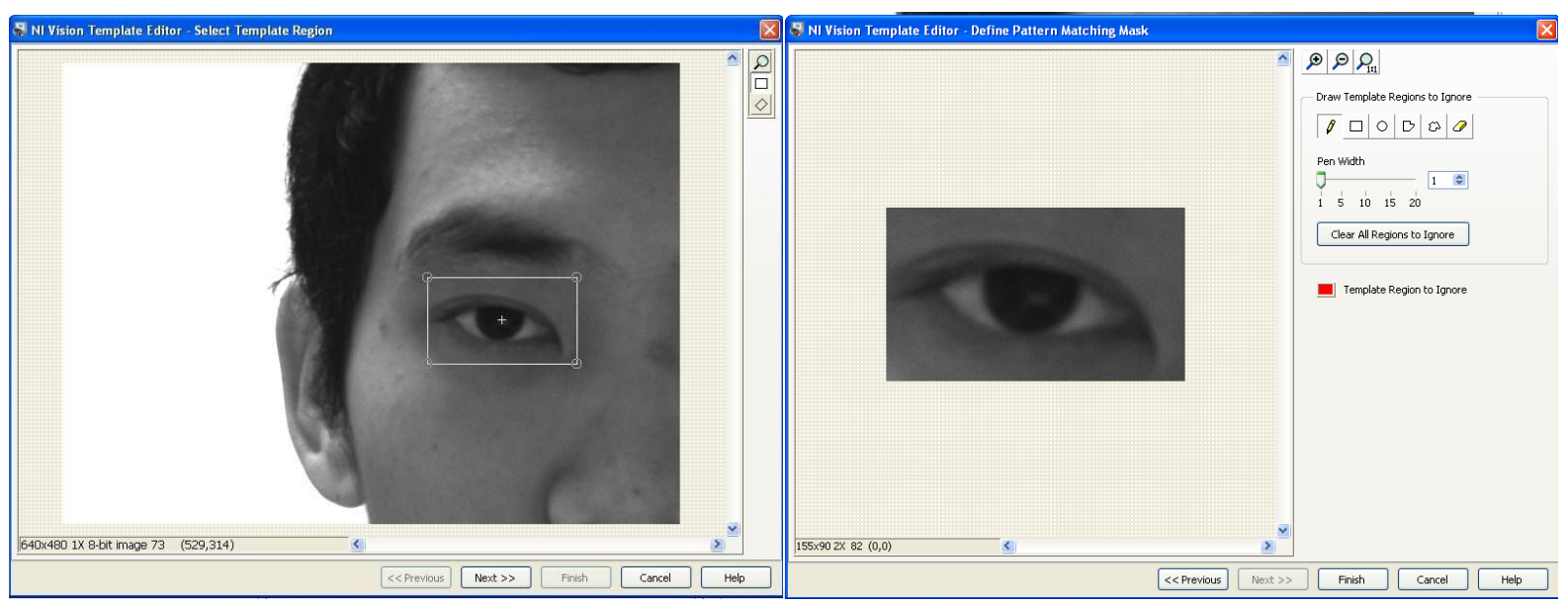

Figure 2. Eye template selection process: First a single frame of the reader's face is captured. Then, the eye area is manually selected by clicking and dragging to cover a rectangular box with the eye to be saved as a template.

Table 1. Steps involved in the real-time iris tracking algorithm

\begin{tabular}{|c|l|}
\hline & \multicolumn{1}{c|}{ Real-Time Iris Tracking } \\
\hline Step 1 & Capture a single image frame to determine and save eye template (non-real-time) \\
\hline Step 2 & Capture a real-time eye image frame using the smart camera interfaced to LabVIEW \\
\hline Step 3 & $\begin{array}{l}\text { Match the eye with the pre-stored eye pattern using normalized cross-correlation (only the } \\
\text { best match is reported) }\end{array}$ \\
\hline Step 4 & $\begin{array}{l}\text { Align the coordinate system and origin with the captured eye image (may be rotated in the } \\
\text { 2D space) }\end{array}$ \\
\hline Step 5 & $\begin{array}{l}\text { Set the Region of Interest to the matching eye area to reduce the processed image area } \\
\text { (surrounding areas are ignored, if there is a good match) }\end{array}$ \\
\hline Step 6 & Calibrate the image to convert pixel coordinates to real-world coordinates \\
\hline Step 7 & $\begin{array}{l}\text { Determine an oval or circle area around the iris based on the points extracted by edge } \\
\text { detection from the Region of Interest }\end{array}$ \\
\hline Step 8 & Calculate iris center coordinates using center of mass \\
\hline Step 9 & Store the coordinates of the iris center in a spreadsheet \\
\hline Step 10 & Repeat Steps 2-9 until the controller stops iris tracking program \\
\hline
\end{tabular}

Figure 3 shows the main module for the iris tracking and recording process based on steps 2-10 of Table 1 . Once the iris center is determined, real-time iris tracking and visualization are achieved by an overlay process that draws a crosshair at the iris center location, and a bounding box around the eye area capturing the iris in the center (Figure 3, 4). Finally, the frame number, $\mathrm{X}$ - and Y-coordinates are written to a spreadsheet on the camera to be retrieved for analysis after real-time data acquisition is complete. Frame number is an indication of the speed of iris tracking, since not all captured frames result in iris detection. The speed of iris tracking depends on the frame rate setting of the camera, speed of iris detection algorithms, and the speed of writing iris center coordinates and frame number to a spreadsheet. The block diagram that shows the functions to write frame number and iris center coordinates to a spreadsheet is shown in Figure 5. The writing process takes about 50 ms resulting in a frame analysis frequency of at most 20 fps which is sufficient for the purposes of this application. 


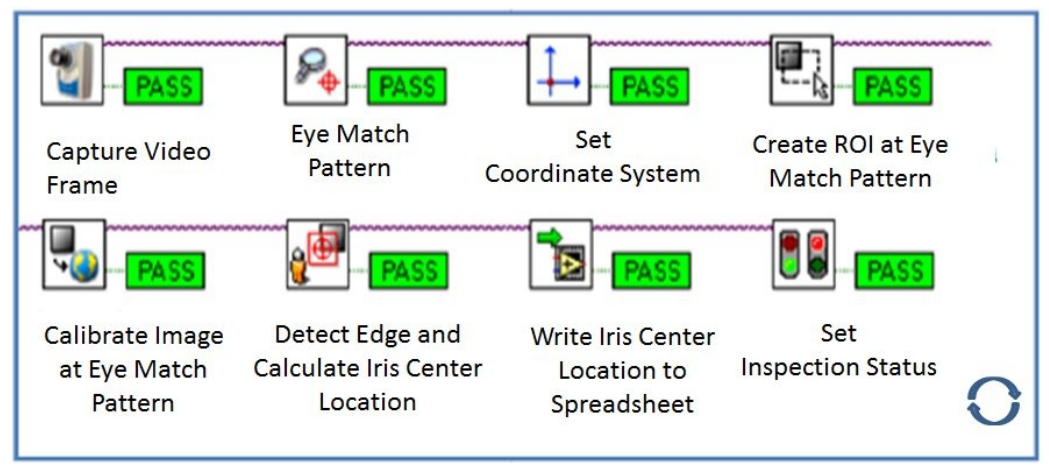

Figure 3. The main LabVIEW module for iris detection and tracking.

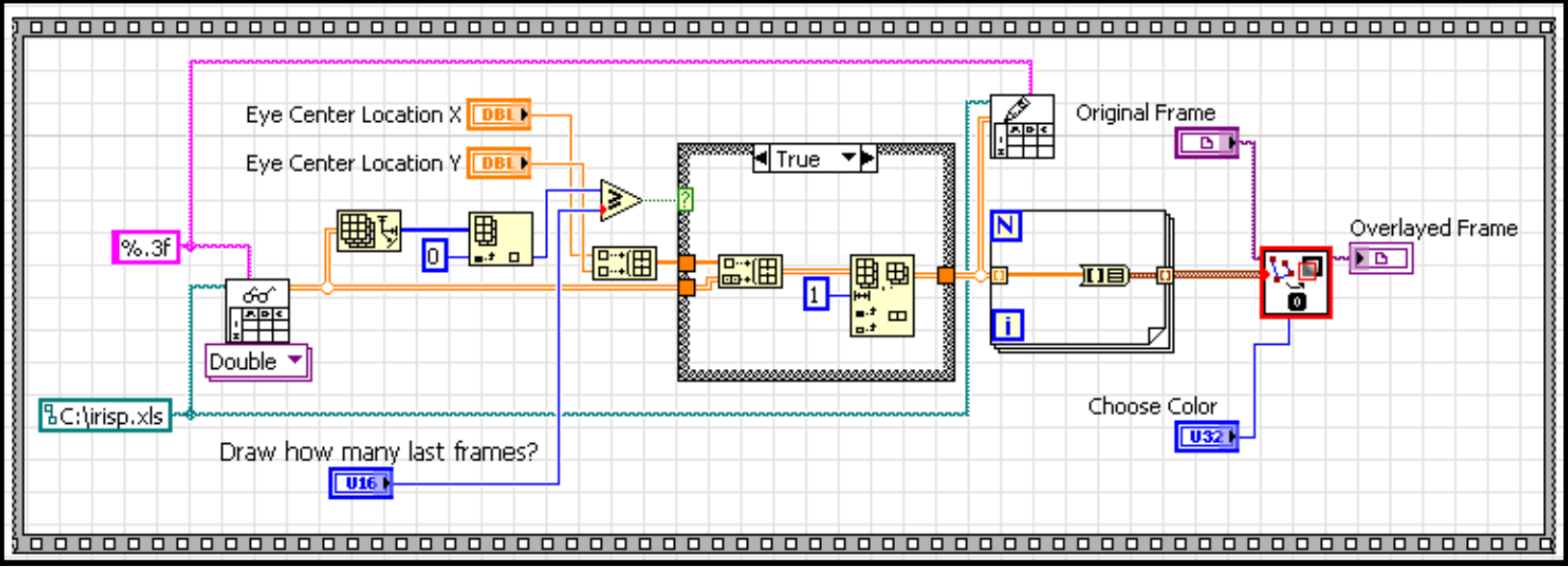

Figure 4. Real-time iris tracking is demonstrated on the screen by a bounding box around the iris center. The bounding box and a cross hair in the center of iris is updated for each frame in which the iris is detected.

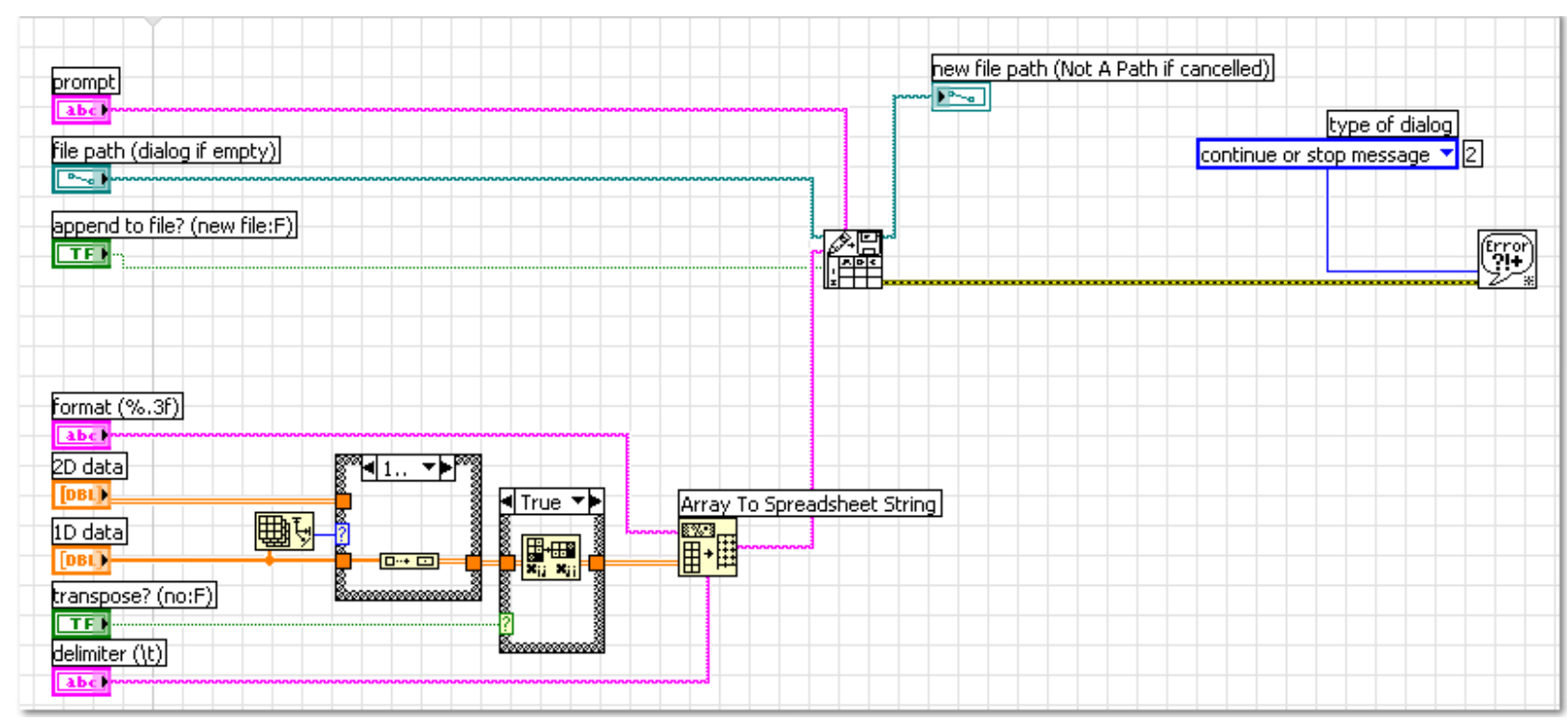

Figure 5. Writing iris center location to file with associated image frame number. 


\section{DATA ACQUISITON PROTOCOL}

The data acquisition process is preceded by informed consent by participants. Each tester is informed of the protocol: "I will first calibrate the camera for you, the reader. First you will look at the center of the screen when prompted. I will then ask you to look on the upper left, upper right, lower right and lower left corners of the screen for about a second each. Then you will view two digital texts. I want you to read the following information on the screen as you normally would read each text. When you are finished with the first one, please say 'I am finished'. I will then open the second text for you to read. When you are finished with the second one, please say 'I am finished'."

Before data acquisition begins, the tester is positioned in front of the camera and the seat is adjusted to the appropriate and comfortable height. The camera is focused and the lens aperture set appropriately to capture a quality image that includes the tester's eye and partial face. Then a single image frame is captured to select the eye template for the tester. Once the tester is ready begin reading, the real-time iris tracking program is started, and reading is preceded by the calibration process where the tester gazes at upper left and right corners and lower right and left corners of the screen for about a second each on cue from the controller to calibrate the viewing area of the screen. The reader continues reading the text after calibration until finished with the text. The controller starts and stops the real-time iris tracking program on a separate computer (PC1, Fig. 1). The tester may be stopped and process repeated, if consistent iris tracking is not achieved during a session.

\section{RESULTS AND ANALYSIS}

Data analysis is performed after the real-time iris tracking is completed. The reading patterns are investigated for the speed of reading determined through the analysis of distances between subsequent data points, how the reader browses through the newspaper text and Web page text by analyzing the iris path, and eye movement in $\mathrm{X}$ and $\mathrm{Y}$ directions.

\subsection{Temporal Analysis of Iris Movement}

Temporal analysis of the iris track provides information about the speed of reading, and offers information about the variations in the direction of the iris track over time. Qualitative analysis of iris path has been performed by plotting the 3D data points (frame number, iris center X-coordinate, iris center Y-coordinate) on a 3D grid to investigate eye movement and variations over time. The frame number begins with a large arbitrary number as stored during real-time iris tracking. The X-coordinates represent the reader's iris' movement in the horizontal direction. The Y-coordinate represents the iris' movement in the vertical direction.

Two distinct set of reading patterns have been observed as shown in Figures 6 and 7.

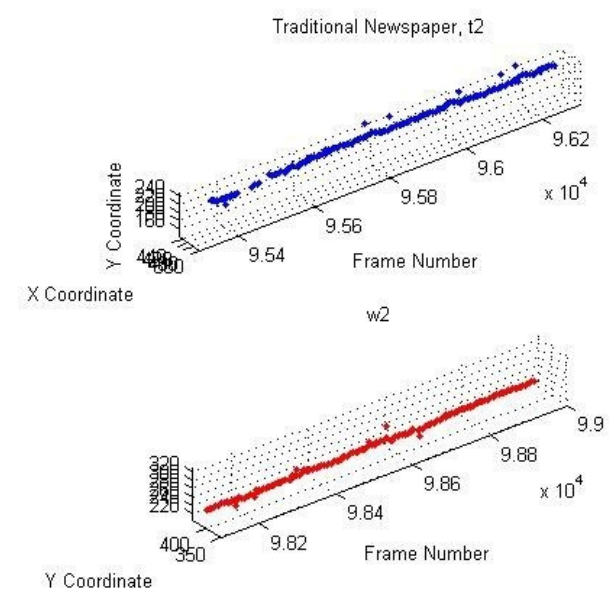

(a)

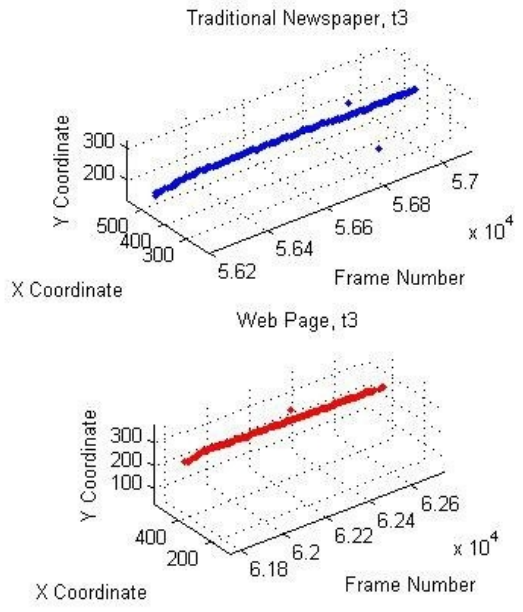

(b)

Figure 6. Smooth reading patterns from two readers (Tester 2 (t2) and Tester $3(\mathrm{t} 3)$ ). The top (blue) represents the traditional newspaper text. The bottom (red) represents the Webpage. 

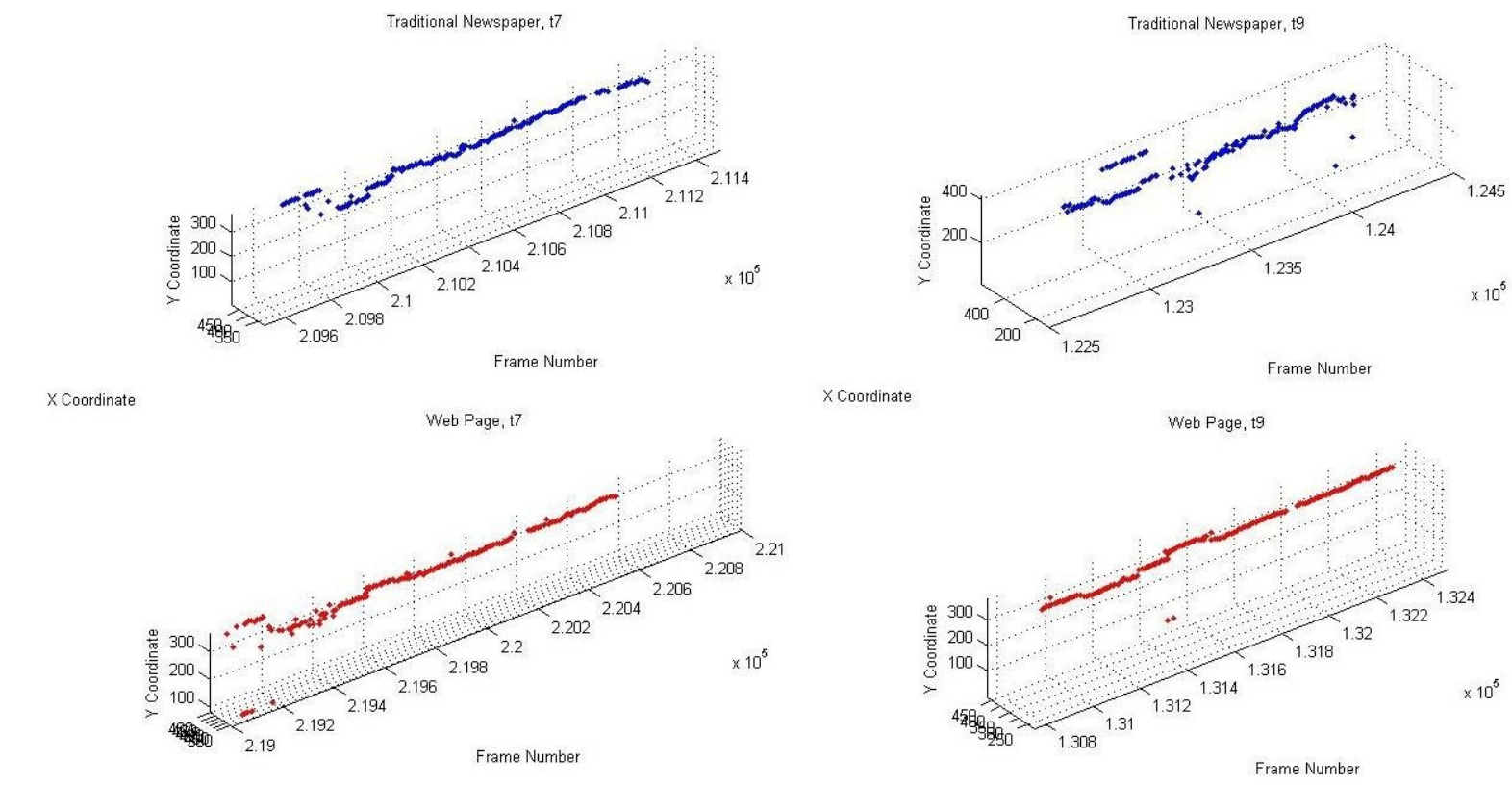

Y. Coordinate

$x$ Coordinate

(a)

(b)

Figure 7. Highly varying reading patterns from two readers (a) Tester 7 (t7) and (b) Tester 9 (t9). The top

(blue) represents the traditional newspaper text. The bottom (red) represents the Webpage.

Figure 6 depicts smooth reading patterns, with some outliers. Figure 7 shows iris tracks with higher variations in $\mathrm{X}$ and $\mathrm{Y}$ iris locations over time. Both figures show that the reading patterns for an individual reader follows similar trends for both regular newspaper text and a Webpage text for news. In the collected set of data, the iris center is stored for every 9 or 10 frames. The frequency of the recorded frames depends not only on the set frame rates, but also the speed at which the data is transferred to the spreadsheet. Currently, this data writing functional block is the largest bottleneck that affects the number of tracked iris locations in a given reading session.

In Figure 8, progression of horizontal motion of the iris in time (across frames) is plotted. This figure demonstrates the iris motion across the page without vertical movement.

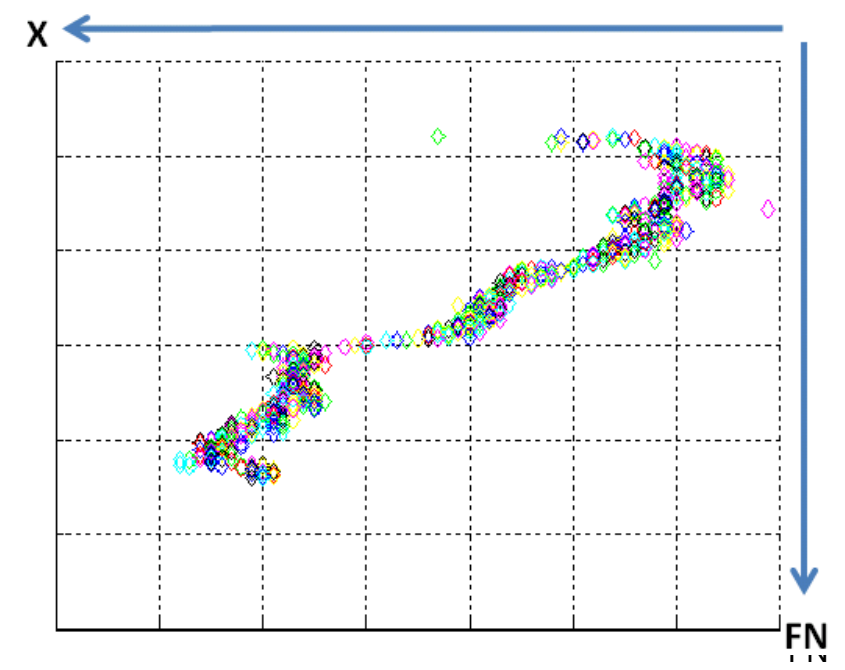

Figure 8. Iris movement in horizontal (X) direction across frames or in time. Note that the positive $\mathrm{X}$ axis is from right to left. 


\subsection{Spatial Analysis of Iris Movement}

Investigation of spatial distribution of the $\mathrm{X}$ - and Y-coordinates of the iris path produces information about the reading/browsing habits, since the iris path represents where the reader is looking on the screen; in particular, when calibrated, XY distribution of iris centers show the area spanned by the iris or read on the page by the tester. For instance, if a tester reads through just text and does not look at any other items on the page such as pictures or links, the iris track matches the spatial composition of the text on the page.

Figures 9 and 10 show examples of 2D iris center distributions from testers that correspond to the smooth and variable reading patterns of Figures 6 and 7, respectively. In Figure 8, the reading patterns are compact, suggesting the tester was looking at a particular region on the screen over time. In Figure 9, the patterns are more spread out, showing higher variance in the iris track. More closely, when the traditional newspaper page (top blue) reading patters are analyzed in Figure 9, it is seen that the iris patterns are confined within a range of twenty points in the horizontal (X) direction, and the same or less in the vertical (Y) direction. For the Webpage news article, the iris pattern range is thirty points or less in the horizontal direction, and twenty points or less in the vertical direction. In contrast, in Figure 10, the reading pattern range jumps to greater than thirty points in both $\mathrm{X}$ and $\mathrm{Y}$ directions, and generally much higher than thirty points, for the two test text types.
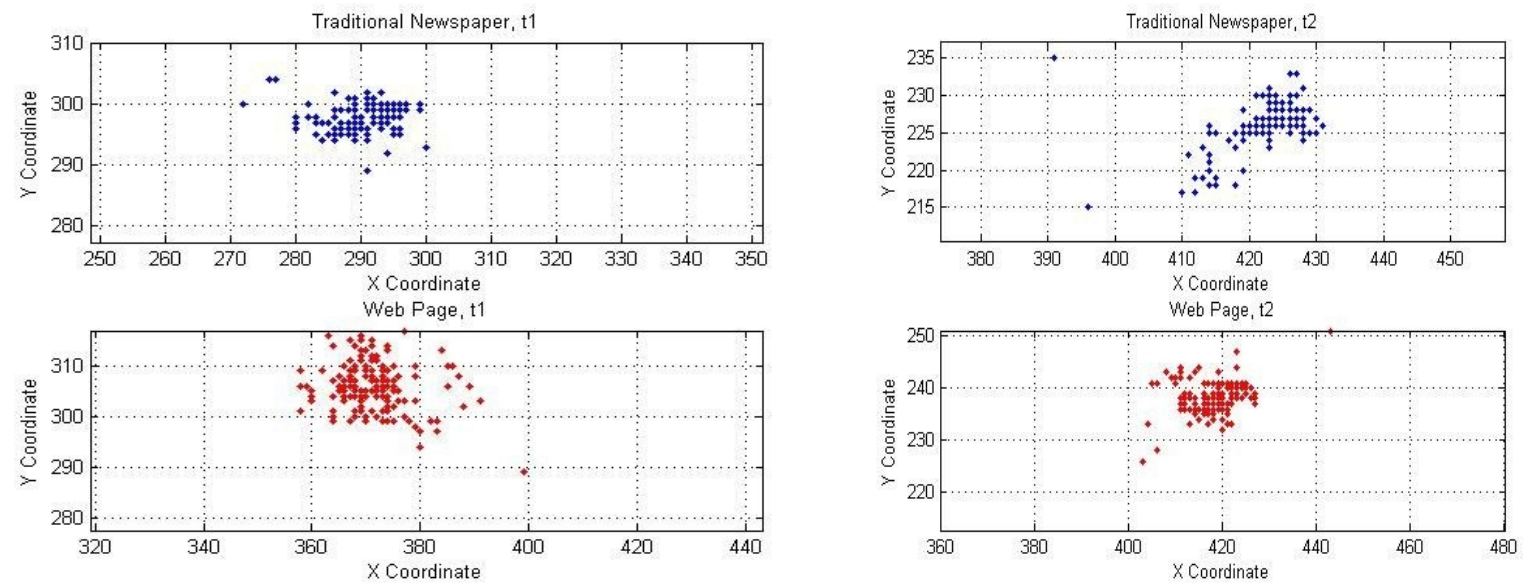

(a)

(b)
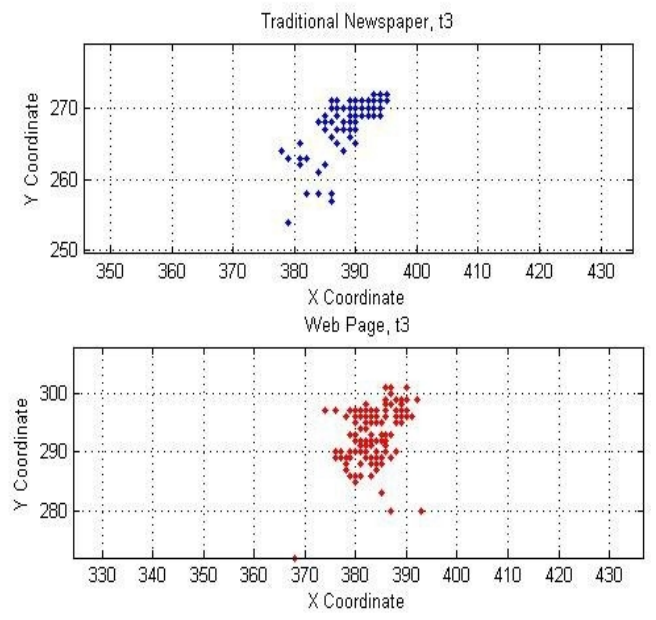

(c)

Figure 9. 2D Reading Patterns for traditional newspaper (top red) and Web Page (bottom blue) for three testers (a) t1, (b) t2, and (c) t3 that correspond to smooth reading patterns of Figure 6. 

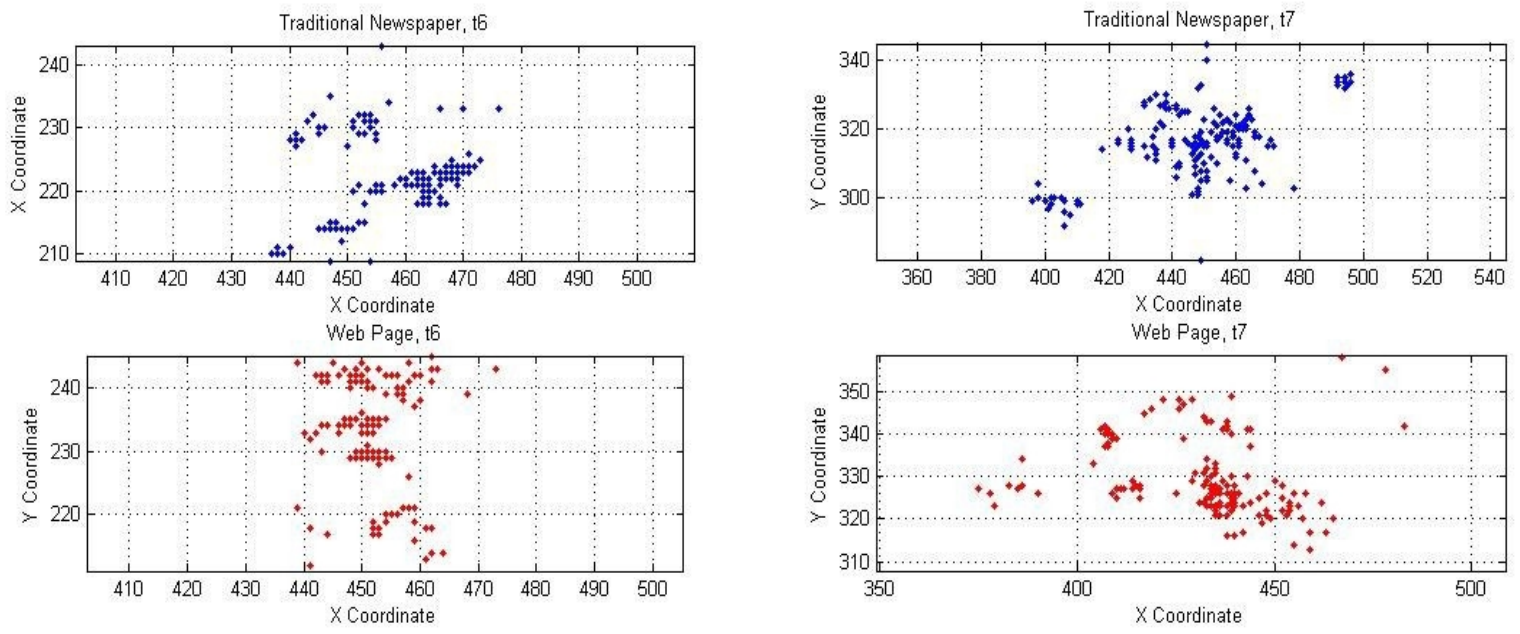

(a)

(b)
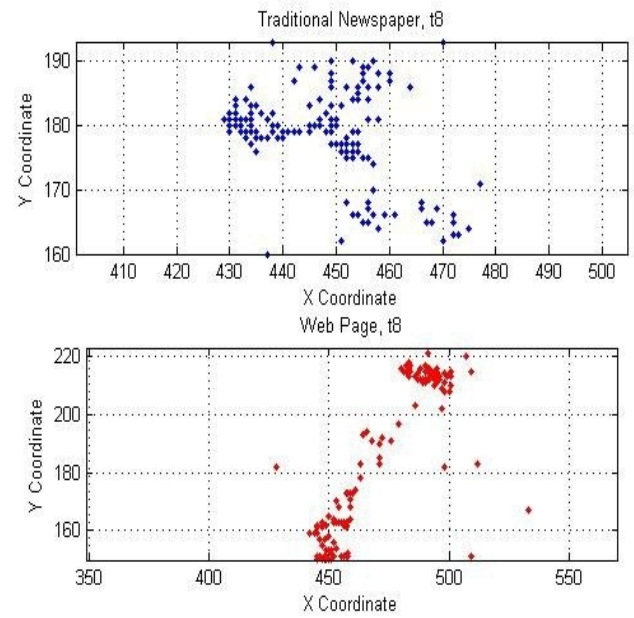

(c)

Figure 10. 2D Reading Patterns for traditional newspaper (top red) and Web Page (bottom blue) for three testers (a) t6, (b) t7, and (c) t8 that correspond to variable reading patterns of Figure 7.

A limitation of the current system involves incomplete calibration for matching the iris track coordinates with the screen coordinates. In the developed iris tracking system, if a reader moves his or her head while reading as opposed to only moving the eyes, the 2D spatial distribution of the of the iris track does not match what is read or looked at on the screen; therefore, readers with significant head movements will produce erroneous interpretations of the iris track. In this study, the real-time iris tracking was recorded by an independent video recorder for analysis and to determine the validity of the iris track. Readers who fidgeted or changed their posture so significantly that affected the relative location of their heads in the camera view have been excluded from analysis.

Besides the head motion, other sources of error include blinking, which could result in either a frame with no match, if the best match is below a preset threshold, or an erroneous match at a different or close by location, such as the eye brows. Missed pattern matches in a given frame could occur due to low normalized cross-correlation scores for detecting the general eye area, or insufficient edge points to detect and compute iris center. In addition, if the frame capture occurs faster than the image processing, then frames will be skipped until image processing on the current 
frame is completed and the algorithm grabs the next available frame. Skipping frames can be tracked by the frame numbers recorded with iris center. Distance of the tester from the camera also affects the accuracy of the iris tracking algorithm, especially if this distance changes during data acquisition. The sources of error must be considered for correct interpretation of the results.

\section{CONCLUSIONS AND FUTURE WORK}

A smart camera has been used for real-time iris tracking to capture reading patterns of readers. Qualitative analysis of iris tracks show two distinct reading patterns among readers. Apparent variations result from smooth patterns that cover a compact reading area, and highly varying patterns covering a larger reading and more scattered area. The preliminary results demonstrate that the reading patterns for an individual reader follows similar trends for both traditional newspaper text and a Webpage text for news with links and other additional information. Spatial information must be calibrated for head movements so that the absolute location of the iris center with respect to the total reading area can be tracked.

The current algorithms do not take head movements into consideration. Because of this, it is not possible to make a oneto-one correlation with the iris track and the area read by the reader. Future work involves gaze tracking or head motion tracking as methods to calibrate the iris center with the absolute location on the reading screen. Experimental data collection can also be improved by normalizing the distance from the camera to the tester.

\section{ACKNOWLEDGMENT}

The authors thank all the participants of the iris tracking project for their time to be testers. The authors also acknowledge the contribution of Peng Su and Ming Teng as controllers, and Eric Trinh in providing the images for this paper. This project has been partially funded by a Texas Research Development Fund (TRDF) grant through Texas A\&M University Office of Research and Graduate Studies.

\section{REFERENCES}

[1] Xiang, B. and Cheng, X., "Eye detection based on improved ad AdaBoost algorithm," Proc. $2^{\text {nd }}$ International Conference on Signal Processing Systems (ICSPS '10), vol. 2, pp. V2-617 - V2-620, 5-7 July 2010.

[2] Xu, G., Wang Y., Li, J., and Zhou, X., "Real time detection of eye corners and iris center from images acquired by usual camera," Proc. Second International Conference on Intelligent Networks and Intelligent Systems, 2009. ICINIS '09, pp. 401-404, 1-3 Nov. 2009.

[3] Sun, X., Chen, G., Zhao. C. and Yang, J., "Gaze Estimation of Human Eye," Proc. 6th International Conference on ITS Telecommunications, pp. 310-313, June 2006.

[4] Hopf, K.; Neumann, F.; Przewozny, D., "Multi-user eye tracking suitable for 3D display applications," Proc. 3DTV Conference: The True Vision - Capture, Transmission and Display of 3D Video (3DTV-CON), pp. 1-4, 16-18 May 2011.

[5] Tan, H., and Zhang, Y., "Detecting eye blink states by tracking iris and eyelids," Pattern Recognition Letters, vol. 27, pp. 667-675.

[6] Xie, X., Sudhakar, R., and Zhuang, H., "Real-time eye feature tracking from a video image sequence using Kalman filter," Southcon/94. Conference Record, pp. 110-115, 29-31 March 1994.

[7] Stasiak, L. and Pacut, A., "Particle filters for multi-face detection and tracking with automatic clustering," Proc. IEEE International Workshop on Imaging Systems and Techniques, IST '07, pp. 1-6, 4-5 May 2007.

[8] Xie, X., Sudhakar, R., and Zhuang, H., "Real-time eye feature tracking from a video image sequence using Kalman filter," IEEE Transactions on Systems, Man and Cybernetics, 25(12), pp. 1568-1577, Dec 1995.

[9] Asano, M., Takano, H., and Nakamura, K., "Eye detection method robust to facial pose changes for eye input device,” 2011 IEEE International Conference on Systems, Man, and Cybernetics (SMC), pp. 602-607, 9-12 Oct. 2011.

[10] Grote-Garcia, S., “College and career readiness,” in Literacy trends and issues: What's hot, J. Cassidy and S. Grote-Garcia (Eds.), Dubuque, IA: Kendall-Hunt, pp. 19-22, 2012. 
[11] The condition of education 2004, Indicator 31: Remedial Coursetaking in National Center for Education Statistics, Section 5-Contexts of PostsecondaryEducation, Washington, DC: U.S. Department of Education, June 2004.

[12] Samuels, S. J., Rasinski, T. V., and Hiebert, E. H., “Eye movements and reading: What teachers need to know,” In What research has to say about reading instruction, S. J. Samuels and A. E. Farstrup (Eds.), Newark, DE: International Reading Association, pp. 25-50, 2011.

[13] Samuels, S. J., Hiebert, E. H., and Rasinski, T. V., "Eye movements make reading possible," in Revisiting silent reading: New directions for teachers and researchers, E. H. Hiebert and D. R. Reutzel (Eds.), Newark, DE: International Reading Association, pp. 24-44, 2010.

[14] Mehrübeoglu, M., Bui, H. T., and McLauchlan, L., "Real-time iris tracking with a smart camera," Proc. SPIE 7871, 787104, 2011. (DOI:10.1117/12.872668)

[15] Mehrubeoglu, M., Pham, L. M., Le, H. T., Ramchander, M., and Ryu, D., "Real-time eye tracking using a smart camera,” Proc. 2011 IEEE Applied Imagery Pattern Recognition Workshop (AIPR ‘11), pp. 1-7, 2011. (doi: 10.1109/AIPR.2011.6176373 ) 